\title{
Futures and Options Exchanges by Time Zones
}

All times given are based on noon Greenwich mean time.

(Note: there will be variations in the times because of the seasonal use of daylight saving)

04.00 Pacific Stock Exchange Vancouver Stock Exchange

06.00 Chicago Board of Trade Chicago Board Options Exchange Chicago Mercantile Exchange Chicago Rice and Cotton Exchange Kansas City Board of Trade MidAmerica Commodity Exchange Minneapolis Grain Exchange Twin Cities Board of Trade Winnipeg Commodity Exchange

07.00 American Stock Exchange Coffee, Sugar and Cocoa Exchange Commodity Exchange Inc. Montreal Exchange New York Cotton Exchange New York Futures Exchange New York Mercantile Exchange New York Stock Exchange Philadelphia Stock Exchange Toronto Futures Exchange

09.00 Bolsa Brasileira de Futuros Bolsa Mercantil \& de Futuros Bolsa de Mercardorias de Sao Paulo

12.00 Baltic Futures Exchange European Mercantile Exchange Irish Futures and Options Exchange International Petroleum Exchange London Options and Futures Exchange London International Financial Futures Exchange London Metal Exchange London Traded Options Market

13.00 Amsterdam Pork and Potatoes Terminal Markets Brussels Optie en Futuresbeurs European Options Exchange Deutsche Terminboerse
Guarantee Fund for Danish Futures and Options Lille Potatoes Futures Market Marché á Terme d'Instruments Financiers Marché des Options Negociables de la Bourse de Paris Mercado Espanol de Futuros Financiers Norwegian Options Market Options Market of France Rotterdam Energy Futures Exchange Spanish Options Market Stockholm Options Market Swiss Options and Futures Market

14.00 Finnish Options Brokers Limited South African Futures Exchange

20.00 Hong Kong Futures Exchange Kuala Lumpur Commodity Exchange Manila International Futures Exchange Singapore International Monetary Exchange

21.00 Hokkaido Grain Exchange Kanmon Commodity Exchange Kobe Grain Exchange Kobe Raw Silk Exchange Kobe Rubber Exchange Maebashi Dried Cocoon Exchange Nagoya Grain and Sugar Exchange Nagoya Stock Exchange Nagoya Textile Exchange Osaka Grain Exchange Osaka Securities Exchange Osaka Sugar Exchange Osaka Textile Exchange Tokyo Commodity Exchange Tokyo Grain Exchange Tokyo International Financial Futures Exchange Tokyo Stock Exchange Tokyo Sugar Exchange Toyohashi Dried Cocoon Exchange Yokohama Raw Silk Exchange

22.00 Australian Stock Exchange Sydney Futures Exchange

24.00 New Zealand Futures Exchange 